\title{
Comparative sequence analysis of the accessory and nucleocapsid genes of feline coronavirus strains isolated from cats diagnosed with effusive feline infectious peritonitis
}

\author{
Supansa Tuanthap ${ }^{1,2} \cdot$ Natdaroon Chiteafea ${ }^{2} \cdot$ Jatuporn Rattanasrisomporn $^{2} \cdot$ Kiattawee Choowongkomon $^{1}$
}

Received: 9 April 2021 / Accepted: 8 June 2021 / Published online: 7 August 2021

(c) The Author(s), under exclusive licence to Springer-Verlag GmbH Austria, part of Springer Nature 2021

\begin{abstract}
Feline infectious peritonitis (FIP) is a lethal infectious disease of domestic cats caused by feline coronavirus (FCoV) infection. Feline infectious peritonitis virus (FIPV) is a mutant type of FCoV that is characterized by causing fibrinous serositis with effusions in the pleural and abdominal cavities (wet form) and/or granulomatous-necrotizing inflammatory lesions in several organs (dry form). There have been numerous studies on FIP worldwide, whereas information about this disease in Thailand is still limited. Most studies involving molecular surveillance and evaluation of FCoV field strains have examined the genetic diversity of the spike and accessory ORF3c coding regions. Of these, the $\mathrm{S}$ gene is more divergent and is responsible for the two FCoV serotypes, while ORF3c harbors mutations that result either in early termination or destruction of the protein. In this study, we investigated the genetic diversity and genetic relationships among the current Thai and global FCoV strains in the accessory and nucleocapsid genes using a virus-specific PCR method. Comparative sequence analysis suggested that the Thai FCoV isolates were most closely related to strains reported in the Netherlands, the USA, and China. In the ORF3ab sequences, some Thai strains were more than $99 \%$ identical to the DF-2 prototype strain. Truncation of the 3a gene product was found in Thai FCoV strains of group 2. Amino acid deletions were observed in the N, ORF3c, and ORF7b proteins of Thai FCoV sequences. The accessory gene sequence divergence may be responsible for driving the periodic emergence and continued persistence of FCoVs in Thai domestic cat populations. Our findings provide updated information about the molecular characteristics of the accessory and nucleocapsid genes of FCoV strains in circulation that were not previously documented in this country.
\end{abstract}

$\begin{array}{ll}\text { Abbreviations } \\ \mathrm{E} & \text { Envelope gene } \\ \text { FCoV } & \text { Feline coronavirus } \\ \mathrm{FIPV} & \text { Feline infectious peritonitis virus } \\ \mathrm{kb} & \text { Kilobase } \\ \mathrm{M} & \text { Membrane gene } \\ \mathrm{N} & \text { Nucleocapsid gene } \\ \text { ORF } & \text { Open reading flame }\end{array}$

Handling Editor: Zhenhai Chen.

Jatuporn Rattanasrisomporn

fvetjpn@ku.ac.th

$\triangle$ Kiattawee Choowongkomon

fsciktc@ku.ac.th

1 Department of Biochemistry, Faculty of Science, Kasetsart University, Bangkok 10900, Thailand

2 Department of Companion Animal Clinical Sciences, Faculty of Veterinary Medicine, Kasetsart University, Bangkok 10900, Thailand

$\begin{array}{ll}\text { S } & \text { Spike gene } \\ \text { TGEV } & \text { Transmissible gastroenteritis virus } \\ \text { UTR } & \text { Untranslated region }\end{array}$

\section{Introduction}

Feline coronavirus (FCoV) is a member of the family Coronaviridae and the genus Alphacoronavirus. It is a single-stranded positive-sense RNA virus with a 5' cap and $3^{\prime}$ poly-A tail. The FCoV genome is approximately 29 kilobases $(\mathrm{kb})$ in length and contains eight genes flanked by a $5^{\prime}$ untranslated region $\left(5^{\prime}\right.$ UTR) and a $3^{\prime}$ UTR (nucleotide positions 311 and 276, respectively). The 5' two-thirds of the genome (nucleotide positions 312 to 20416) encodes the replicase protein, which is associated with RNA replication and transcription [1]. Four structural genes are located downstream, encoding the spike (S) (nucleotide positions 20,413 to 24,810), envelope (E) (nucleotide positions 25,897 to 26,145 ), membrane (M) 
(nucleotide positions 26,156 to 26,947 ), and nucleoprotein (N) (nucleotide positions 26,960 to 28,087 ) proteins. Two ORFs encode major accessory proteins: ORF3, located between the $\mathrm{S}$ and $\mathrm{E}$ genes, encodes the proteins $3 \mathrm{a}, 3 \mathrm{~b}$, and $3 \mathrm{c}$ (nucleotide positions 24,822 to 25,910 ), and ORF7, located downstream of the $\mathrm{N}$ gene, encodes the proteins $7 \mathrm{a}$ and $7 \mathrm{~b}$ (nucleotide positions 28,092 to 29,022 ). The S protein is responsible for viral receptor binding and induces fusion of viral and cellular membranes during viral entry $[2,3]$. The $\mathrm{E}, \mathrm{M}$, and $\mathrm{N}$ proteins are associated with the viral RNA. The accessory genes are important in virulence or viral tropism [4]. The function of the accessory proteins $7 \mathrm{a}$ and $7 \mathrm{~b}$ is not fully understood, but previous reports have suggested that both proteins are involved in the immune response to FCoV infection [5]. Accordingly, the 7 a protein is a type I interferon (INF-1) antagonist, while the $7 \mathrm{~b}$ protein induces antibody responses in naturally infected cats. In experimental infections, FIPV mutants that lacked $7 \mathrm{a}, 7 \mathrm{~b}$, or both proteins had lower virulence than the wild-type virus and did not lose their virulence in cell lines [5-9].

Based on S gene sequence diversity, the widely accepted classification system for $\mathrm{FCoV}$ divides virus isolates into two serotypes: FCoV serotype I (FCoV I) and serotype II (FCoV II). FCoV I is a strictly feline type and has been reported to be the predominant serotype causing infections, whereas FCoV II originated from the natural combination between FCoV I, canine coronavirus $(\mathrm{CCoV})$, and transmissible gastroenteritis virus (TGEV) [10, 11]. The $\mathrm{S}$ gene of $\mathrm{FCoV}$ type II originated from $\mathrm{CCoV}$, and this type is less frequently identified than type I [12-15]. Both serotypes are subdivided into two distinct biotypes (also referred to as pathotypes), referred to as feline enteric coronavirus (FECV) and feline infectious peritonitis virus (FIPV).

The FECV biotype only replicates within the intestinal epithelium, causing subclinical infection or mild enteritis $[16,17]$. On the other hand, FIPV is able to infect monocytes and macrophages, leading to the development of immunemediated responses [3, 18, 19]. Genome mutations in FECV that cause it to become highly pathogenic and cause FIP have been estimated to occur in approximately $5 \%$ of persistently infected cats [20-22].

Several previous studies have suggested that the pathotypic switching in natural populations might be associated with accessory genes such as ORF3c or ORF7b and structural genes such as the nucleocapsid gene [23-25]. Moreover, the prevalence and molecular characteristics of FCoV in Thailand have rarely been documented. In the present study, the accessory gene sequences and nearly complete $\mathrm{N}$ gene sequences of Thai FCoV isolates obtained during 2017-2020 were determined to assess their genetic diversity and their relationships to FCoV strains.

\section{Materials and methods}

\section{Criteria for diagnosis of FIP with effusion fluid (wet form)}

The veterinary practitioner used a combination of different types of information to guide a conclusive diagnosis of FIP with effusion in cats. This included physical examination, history taking (to consider the risk of infection), thoracic and abdominal radiography images (or ultrasonography), hematological and serum biochemical profiles, and cytology evaluation of body effusion. Anorexia, weight loss, lethargy, and fever usually occur in sick cats, but the specified criteria for effusive FIP (FIP wet form) were used: abdominal enlargement, an icterus (might or might not present), dyspnea or tachypnea, radiography (or ultrasonography) showing fluid accumulation within thoracic and/or abdominal cavities, abnormal serum biochemical parameters related to a high serum amyloid A (SAA) value $(>8 \mathrm{mg} / \mathrm{L})$ and total serum protein (TP) value above the cutoff value ( $>8 \mathrm{~g} /$ $\mathrm{dL}$ ), hypoalbuminemia, hyperglobulinemia, and an albu$\mathrm{min} /$ globulin $(\mathrm{A}: \mathrm{G})$ ratio ranging from $<0.4$ to 0.8 . The hematological abnormalities mostly correlated with anemia, neutrophilia, and lymphopenia. Body effusions were clear, viscous/sticky, straw-yellow, and protein-rich (total protein concentration in exudate, $>35 \mathrm{~g} / \mathrm{l}$ ). Macroscopic and cytological evaluation of effusion showed low cell counts (usually fewer than $5 \times 10^{9}$ cells/ $\mathrm{L}$ in transudate) with inflammatory cells (typically macrophages and neutrophils). Effusion smears containing pink protein granules (thick eosinophilic proteinaceous backgrounds) [26].

Although Rivalta's test and immunohistochemistry (IHC) (to detected FCoV antigen in affected tissues) were not performed for FIP diagnosis, other diseases associated with extravasation of protein-rich fluid (such as non-septic peritonitis, modified transudate) were also examined to avoid misdiagnosing FIP in unaffected cats. Cats with the non-effusive form of FIP (dry form), which does not produce body cavity effusion, were excluded from this study.

According to the specified criteria for effusive FIP diagnosis in this study, 67 cats with suspected FIP that were brought to the Veterinary Teaching Hospital, Kasetsart University, Bangkok, Thailand, from August 2017 to August 2020 were included in the study. Most of the cats were from the Bangkok metropolitan region (Supplementary Fig. S1). All of the cats were privately owned and living in households with or without other cats. None of the cats had been vaccinated against FIP, because application of the FIP vaccine was not a common practice in pet clinics. Information about the clinical severity, common vaccination history, and blood profiles were recorded. Information for all FIPsuspected cats is listed in Supplementary Table S1. 


\section{Sample collection}

Pleural and/or peritoneal fluid samples $(n=62)$, postmortem tissue $(n=1)$, plasma samples $(n=3)$, and fecal swabs $(n=3)$ were collected from 67 cats with suspected FIP. The cats were sedated for collection of body effusions.

\section{Sample preparation, RNA extraction, and CDNA synthesis}

Three-hundred $\mathrm{ml}$ of body effusion samples and suspensions of rectal swab samples from FIP-suspected cases were prepared as $10 \%(\mathrm{w} / \mathrm{v})$ suspensions with sterile phosphatebuffered saline. The fluid was clarified by centrifugation at $1,000 \mathrm{~g}$ for $10 \mathrm{~min}$, and the supernatant was collected [27]. Viral RNA was extracted using a viral RNA purification kit according to the manufacturer's instructions (E.Z.N.A. Viral RNA Kit, Omega Bio-tek, GA, USA). Five ml of RNA-containing sample was added to the premix of the RevertAid First Strand cDNA Synthesis Kit (Thermo Scientific Inc, MA, USA) for reverse transcription (cDNA synthesis) with random hexamers, following the manufacturer's instructions.

\section{Viral nucleic acid detection and sequence analysis}

Sample cDNA was initially tested for the highly conserved 3'untranslated region (3'-UTR) of type I and type II FCoV (sense, P205: 5'-GGCAACCCGATGTTTAAA ACTGG-3'; antisense, P211: 5'-CACTAGATCCAGACG TTAGCTC-3') (targeting a 223-bp region) using Phusion Hot Start II High-Fidelity DNA Polymerase (Thermo Scientific Inc., MA, USA) [19]. Cycling parameters were as follows: initial denaturation at $95{ }^{\circ} \mathrm{C}$ for $2 \mathrm{~min}, 35$ cycles of denaturation at $94{ }^{\circ} \mathrm{C}$ for $30 \mathrm{~s}$, annealing at $60{ }^{\circ} \mathrm{C}$ for $30 \mathrm{~s}$, and extension at $72{ }^{\circ} \mathrm{C}$ for $1 \mathrm{~min}$, and a final extension at $72{ }^{\circ} \mathrm{C}$ for $5 \mathrm{~min}$. All positive samples were subsequently subjected to partial amplification of gene segments encoding the $3 \mathrm{a}, 3 \mathrm{~b}, 3 \mathrm{c}, \mathrm{E}, \mathrm{M}, \mathrm{N}, 7 \mathrm{a}$, and $7 \mathrm{~b}$ proteins, which are FCoV type II-specific. The specific primers used to amplify the ORF3 (3a, 3b and 3c) region were sense (5'-TAAAATGGCCKTGGTATGTGT-3') and antisense (5-ACTTCTCATRAACGGTGCAG- $3^{\prime}$ ), those for the E to $\mathrm{M}$ region were sense (5'-GCTGCACCGTTTATGAGA AG-3') and antisense (5'-ACCACCATG ATTTGGTCC TTC-3'), those for the $\mathrm{N}$ region were sense (5'-CGTCAA CTGGGGAGATGAAC-3') and antisense (5'-CATCTC AACCTGTGTGTCATC-3'), and those for the ORF7 (7a and $7 \mathrm{~b}$ ) region were sense (5'-GTG TTT GATGACACA CAGGTTG- $3^{\prime}$ ) and antisense (5'-TTGGCTCGTCATAGC GGATC-3'). The primer sequence details, including positions and amplicons sizes, are shown in Table 1. The PCR cycling parameters for these primer sets were as follows: initial denaturation at $95{ }^{\circ} \mathrm{C}$ for $2 \mathrm{~min}, 35$ cycles of denaturation at $94{ }^{\circ} \mathrm{C}$ for $30 \mathrm{~s}$, annealing at $65^{\circ} \mathrm{C}$ for $30 \mathrm{~s}$ (all primer pairs had the same annealing temperature), and extension at $72{ }^{\circ} \mathrm{C}$ for $90 \mathrm{~s}$, and a final extension at $72{ }^{\circ} \mathrm{C}$ for $5 \mathrm{~min}$. PCR amplicons were resolved and purified using agarose gel electrophoresis. Nucleotide sequences were determined by the Sanger method. Sequences were edited manually in EditSeq (DNASTAR, Madison, USA). Multiple sequence alignments were performed with ClustalW 2.0 software. Phylogenetic trees were constructed by the maximum-likelihood method with 1,000 bootstrap replicates, using MEGA6 software [28]. The best-fit models were determined using the model test parameter. Bootstrap values $\geq 80 \%$ were considered significant. The sequences of the prototypic or vaccine strain sequences WSU 79-1146 (AY994055) and DF-2 (DQ286389.1) were included in all phylogenetic trees. Residue position numbering was based on WSU 79-1146.

Nucleotide sequences were deposited in the GenBank database under the accession numbers MW545831MW545861 for ORF3a, MW545862-MW545891 for ORF3b, MW558570- MW558578 for ORF3c, MW545893MW545901 for E, MW545902- MW545910 for partial M, MW558579- MW558584 and MW558586 for N, MW558587- MW558595 for ORF7a, and MW558596MW558605 for ORF7b.
Table 1 Oligonucleotide primers used in this study

\begin{tabular}{lllllc}
\hline Gene & Name & Sequence & Position & bp & Product size \\
\hline ORF3abc & FIP-F7 & TAAAATGGCCKTGGTATGTGT & $24628-24648$ & 21 & \\
(DQ848678) & FIP-R7Sc & ACTTCTCATRAACGGTGCAG & $25271-25291$ & 20 & $997 \mathrm{bp}$ \\
E and M & FIP-F8 & GCTGCACCGTTTATGAGAAG & $5254-5270$ & 20 & \\
(FJ917523.1) & FIP-R8 & ACCACCATGATTTGGTCCTTC & $6232-6252$ & 21 & 999 bp \\
N & FIP-F9N & CGTCAACTGGGGAGATGAAC & $6632-6651$ & 20 & \\
(FJ917523.1) & FIP-R9N & CATCTCAACCTGTGTGTCATC & $7698-7718$ & 21 & \multirow{2}{*}{1087 bp } \\
ORF7ab & FIP-F10 & GTGTTTGATGACACACAGGTTG & $7692-7713$ & 22 & \\
(FJ917523.1) & FIP-R10 & TTGGCTCGTCATAGCGGATC & $8848-8867$ & 20 & 1176 bp \\
\hline
\end{tabular}




\section{Results}

Thirty-nine samples from cats with suspected infection tested positive for the $3^{\prime}$ UTR region. From 31 of the 39 samples, the full-length $3 \mathrm{a}$ and $3 \mathrm{~b}$ genes were successfully sequenced, from nine samples, the $3 \mathrm{c}$ and $7 \mathrm{a}$ genes were successfully sequenced, from eight samples, the E gene was successfully sequenced, from seven samples, the partial $\mathrm{N}$ gene was successfully sequenced, and from 10 samples, the $7 \mathrm{~b}$ gene was successfully sequenced.

\section{Analysis of the ORF3a and $3 \mathrm{~b}$ gene region}

In phylogenetic analysis, the Thai FCoV strains diverged and branched as two separate groups. Twelve Thai strains either clustered with previously identified strains from different countries or with prototypic FIPV strains (WSU 79-1146 and WSU 79-1683) (termed the G1 group). The rest of the 19 Thai strains grouped in the same clade including the prototypic strain DF-2 (termed the G2 group) (Fig. 1).

FCoV strains identified in the Netherlands, China, and the USA from 2006 to 2008 had a close genetic relationship to the Thai G1 group in both gene segments.

\section{Amino acid sequence alignment}

\section{Analysis of ORF3a gene region}

Amino acid sequence alignments were made for positions 8108-8177 (nucleotide positions 24,324-24,531) for G1 and positions 8108-8138 (nucleotide positions 24,324-24,414) for G2. Nucleotide position 24,980 of Thai G2 strains is $\mathrm{T}$ instead of $\mathrm{A}$, resulting in a premature stop codon when compared to Thai G1 strains (Supplementary Figure S2). Sequence analysis of ORF3a revealed that the Thai G1 strains shared very low relatedness to members of the G2 group at the amino acid sequence level, whereas Thai G2 strain sequences were identical to that of the DF-2 prototypic strain (100\% amino acid sequence identity). Both Thai groups shared low sequence similarity with the WSU $79-1146$ prototypic strain $(47.7-60.4 \%$ and $78.68 \%$ identity, respectively).

\section{Analysis of ORF3b gene region}

Amino acid sequence alignments were made for positions 8338-8311 (nucleotide positions $25,014-24,933$ ) for G1 and positions 8388-8427 (nucleotide positions 25,16425,281 ) for G2. The ORF3b sequence of both Thai groups was shorter than that of the prototype without a frameshift. The members of the Thai $\mathrm{G} 2$ group are genetically close to transmissible gastroenteritis virus (TGEV) (95.60\% amino acid sequence identity) and their amino acid sequence is identical to that of the DF-2 strain.

Sequences of eight Thai strains of the G1 group (KUTT, KU01, KU12, KU30, KU33, KU53, KU57 and KU60) and one Thai strain (KU72) of the G2 group spanned the full length of the ORF3c gene, $\mathrm{E}$ gene, and partial $\mathrm{M}$ gene. These sequences were therefore used for further analyses.

\section{Analysis of ORF3c gene region}

Most of the Thai strains (8/9) (KUTT, KU01, KU12, KU30, KU33, HU53, KU57 and KU60) belonged to the same lineage as FCoV strains from other countries. Strains identified from China, the USA, the Netherlands, and Belgium shared the highest sequence similarity to these Thai strains. Only one Thai strain (KU72) clustered within the prototypic FCoV strains (WSU 79-1146, WSU 79-1683 and DF-2), $\mathrm{CCoV}$, and members of the TGEV lineage. KU72 was more closely related to the DF-2 prototypic strain $(92.70 \%)$.

Alignment of amino acid sequences encompassing positions 8230 to 8475 (nucleotide positions 24,692-25,426) showed that ORF3c and had a shortened sequence without a frameshift when compared to the prototype. The Thai strain KU12 had nine missing residues at positions 8396-8405.

\section{Analysis of $E$ and partial $M$ gene regions}

The phylogenetic tree showed that eight of nine Thai strains (KU12, KUTT, KU01, KU57, KU60, KU53, KU30 and KU33) (KUTT, KU01, KU12, KU30, KU33, KU53, KU57 and KU60) belonged to the same lineage as previously identified strains from other countries and were most closely related to FCoVs identified in China and Belgium. Only one Thai strain, KU72, was distinct from other Thai FCoV strains and shared high genetic relatedness to DF-2 (95.64\% identity) and WSU79-1146 (90.46\% identity).

Partial amino acid sequences of the $\mathrm{M}$ gene were aligned from amino acid positions 8637 to 8753 (nucleotide positions 25,644-26,251). Residue deletions in this region relative to WSU79-1146 were observed at position 8672 in KU72, KU12, and KU01 KU01, KU12 and KU72.

\section{Analysis of $\mathbf{N}$ gene region}

Most of the Thai FCoV strains (6/7) belonged to the same lineage as FCoV strains identified previously in other countries. The Thai strain KU62 was genetically distinct from the others and formed a separate branch with prototypic strains (DF-2 and WSU 79-1146) and canine CoV (CCoV) strain 171. KU62 shared a high genetic relatedness to prototypic strains (94.05-94.12\%) and the CCoV strain 171 (94.04\%). 
Fig. 1 Phylogenetic tree of the FCoV ORF3ab gene. The black dots in front of the names represent Thai $\mathrm{FCoV}$ isolates from this study, bold with underlining indicates FCoV type I, and FCoV prototypic strains are shown in red. TGEV, transmissible gastroenteritis virus; $\mathrm{CCoV}$, canine coronavirus

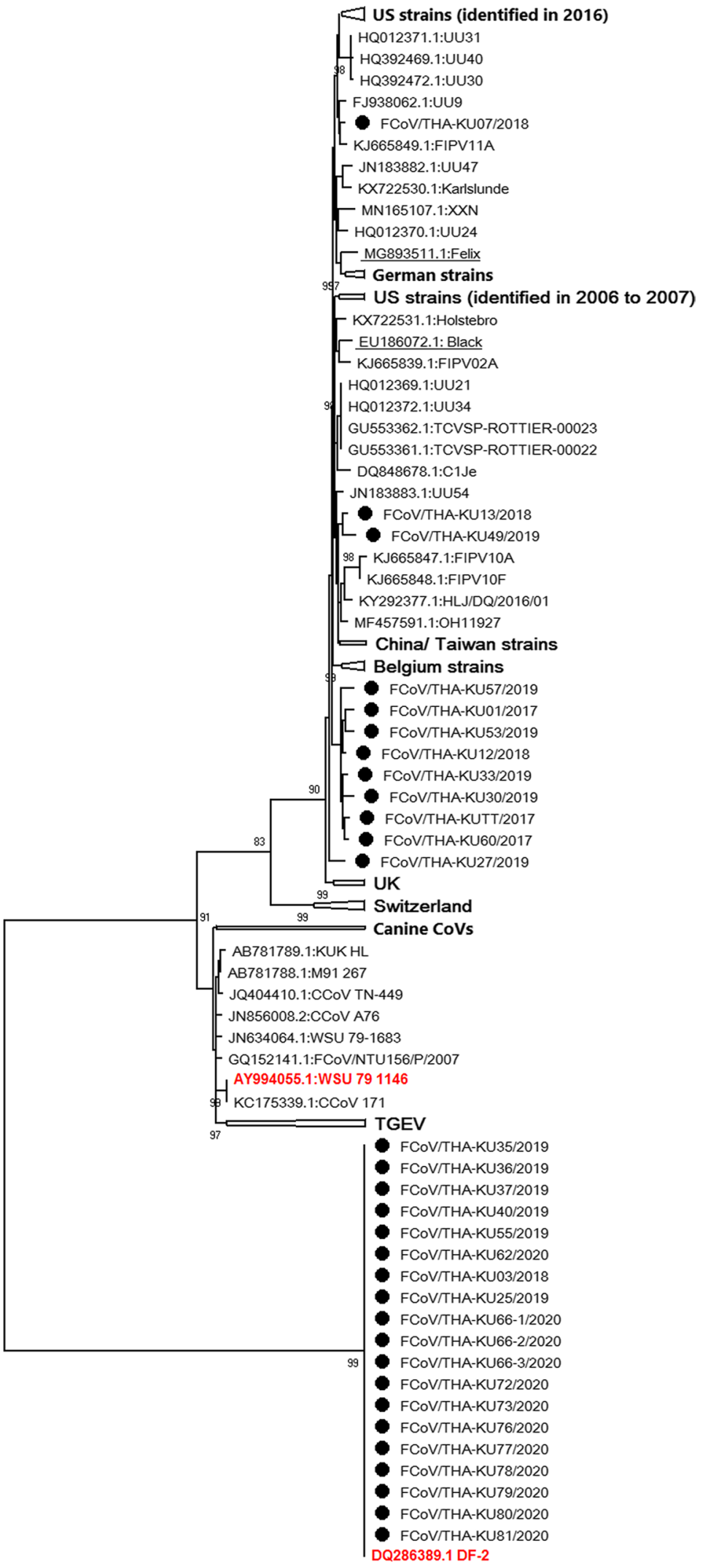


A sequence alignment encompassing positions 8941-9256 with WSU 79-1146 (nucleotide positions 26,820-27,770) revealed deletions in the middle of the $\mathrm{N}$ protein at positions 9089-9090 of isolate KUTT, positions 9106-9107 of isolate KU30, and 9118-9120 of isolate KU12.

\section{Analysis of the ORF7a gene region}

All Thai strains (KUTT, KU03, KU12, KU30, KU33, KU55, KU62, KU77 and KU78) belonged to the same lineage as FCoV strains identified previously in other countries. Some Thai strains shared an identical sequence (KU30 and KU33) (KU03 and KU77) with each other. There were no amino acid insertions or deletions at positions 9280-9382 (nucleotide positions $27,842-28,147$ ) in any of the Thai strains.

\section{Analysis of the ORF7b gene region}

Phylogenetic analysis revealed that 10 Thai strains were divided into two subgroups (Supplementary Fig. S3). Seven strains (KU12, KU30, KU33, KU55, KU62, KU76, KU78) belonged to a lineage including FCoV strains from China, the USA (in 2016), Japan, and the Netherlands (UU54), while the remaining three Thai strains (KU03, KU-TT and KU77) formed a cluster with FCoV I strains and Belgian strains. The prototypic strain (WSU 79-1146) was genetically distinct from all Thai subgroups.

An alignment of amino acid sequences encompassing positions 9384-9590 (nucleotide positions 28,152-28,770) revealed missing residues at position 9392 in three Thai FCoV sequences (KU30, KU33 and KU78) (Supplementary Fig. S4). On the other hand, the amino acid sequences of KU30 and KU33 were identical.

A world map showing the countries of origin of the reference FCoV strains is shown in Supplementary Figure S5.

\section{Discussion}

Antemortem diagnosis of FIP in clinical practice remains difficult because clinical findings are varied and non-specific; neither complete blood counts (CBC) nor serum biochemical parameter changes are able to discriminate between FIP and non-FIP cases. Moreover, the A/G ratio reported previously differs from the optimum critical value [29-35]. FIP is suspected when at least four of the following criteria are met: abnormal clinical signs (with abdominal enlargement), hematological and biochemical profile changes, diagnostic imaging (X-rays or ultrasound examinations) showing effusion fluid accumulation within the thoracic and/or abdominal cavity, a positive PCR result (FCoV detection), positive Rivalta's test, and FIP antigen identified in affected tissue by histopathology [26, 36]. The definitive diagnosis of
FIP is immunohistochemical staining (IHC) (gold standard test). However, IHC is a postmortem method. To support an antemortem diagnosis, we applied PCR to detect FCoV RNA in specimens (effusion, fecal, and plasma samples) collected from live cats with suspected effusive FIP. An FIP diagnosis was made when samples were positive in a PCR assay targeting the $3^{\prime} \mathrm{UTR}$ and ORF3ab regions. The definitive diagnosis for FIPV followed the sequence analysis results.

Since the latest report on circulating FCoV in Thailand in 2012, the continuous epidemiological study of Thai FCoV has been very limited [37]. The fact that new FIP-suspected cases have continually appeared at our Veterinary Teaching Hospital and that cats have required multiple treatments for FIP-like symptoms indicates that FCoV remains prevalent within Thai cat populations [33].

Our multi-year study provides additional information about the diversity of FCoV. The findings indicate that Thai FCoV strains generally cluster within the clade of global FCoVs, supporting the close genetic relationship among their ancestors. The Thai FCoVs were the most closely related to strains identified in the USA between 2006 and 2008 for $3 a$, the Netherlands for $3 b$, China for $3 c$ and $7 b$, Belgium for $\mathrm{E}$ to partial M, Italy for N, and Taiwan for $7 \mathrm{a}$.

Animal movement appears to be responsible for the distribution of FCoV and its introduction into other countries. In 1969, FCoV was first discovered in Canada, and it was subsequently introduced into Germany and Europe in the 1970s. After that, the disease became pandemic [38]. The immigration of American soldiers and their families (together with their pets, including cats) into Germany and Japan after World War II has been hypothesized to be the cause of the spread of FCoV in those countries. The great increase in the number of cats may have facilitated virus transmission in those areas where the virus was not previously reported [39-41]. Currently, animal transport and trade for breeding purposes (especially purebred cats) or relocation of cat owners to different areas may serve as a mechanism for the spread of the virus.

$\mathrm{FCoV}$ is an RNA virus and is susceptible to mutations in different regions of its genome. In this study, we found deletions in the ORF3c, ORF7b, M, and N gene sequences, and this observation was reproducible when the RNA extraction, amplification, and sequencing were repeated. Previous studies have suggested that the spread of FCoV may have been related to natural mutations in the ORF3c, ORF7b, and S genes [9, 23, 42-45]. Some deletions in the genes encoding nonstructural proteins cause internal frameshifts that can cause protein truncations. Mutations in the ORF3abc and ORF7ab proteins are believed to be the cause of FECV-FIPV switching, particularly in the ORF3c gene $[5,42,46]$. However, mutations in ORF3c are not always involved in FIPV switching, because healthy cats can also carry mutated $3 \mathrm{c}$ 
[24, 44, 47]. Our sequencing studies found hypervariable amino acid positions at many sites in the ORF3abc of the Thai isolates, including internal stop codons. For example, at nucleotide position 24,979-24,981 (ORF3a region), the Thai G2 FCoVs have TAA, while the Thai G1 isolates have TTC or CTC, and the reference FCoV strains have GAC. The introduction of a stop codon leads to premature termination of translation, resulting in a shorter amino acid sequence in the Thai G2 isolates than in the Thai G1 isolates and reference FCoV strains (Supplementary Fig. S6). Further sequencing studies are needed to access the frequency of point mutations, recombination events, and deletions in cats with FIP.

The Thai FCoV isolates exhibited sequence differences that contributed to their clustering into two distinct groups. The analysis of the entire ORF3 gene ( $3 \mathrm{abc}$ ) revealed that the Thai strain KU72 is more closely related to the DF-2 prototype than other Thai FCoVs. Although we were not able to sequence the ORF 3 c region of all the Thai G2 strains, it is probable that this region has high sequence variability [47]. Apart from 3c, residue deletions in the middle of the $\mathrm{N}$ protein (KUTT, KU12, AND KU30) and in the $7 \mathrm{~b}$ gene (KU30, KU33 and KU78) were also observed. An internal stop codon or deletion in the $7 \mathrm{~b}$ gene indicated the possibility of viruses losing their virulence in vivo $[6,8,48]$, but we were not able to link these variations with disease severity in the FIPV-diagnosed cats.

Some of the Thai FCoV had identical sequences in some parts of the genome but differed in others. It is possible that multiple virus strains have circulated and persisted within Thai domestic cat populations.

The analysis of accessory gene sequences indicated that the Thai FCoV isolates are phylogenetically distant from the prototypic and vaccine strains (WSU 79-1146 and DF-2). In contrast to the $\mathrm{E}$ and $\mathrm{M}$ gene sequences, the accessory gene sequences of the Thai FCoV isolates belong to the same cluster as the prototypes. The WSU 79-1146 and DF-2 strains have been used in licensed attenuated vaccines, and amino acid differences might be reflected in vaccine effectiveness. Although the accessory genes are believed to affect virulence and viral tropism, the use of accessory gene analysis alone may not accurately predict vaccine potency.

In general, we found that cats of different ages were symptomatic for FIPV infection, including kittens, young cats, and old cats). FCoV-positive cases could be detected throughout the year, suggesting that season does not clearly influence the disease. In this study, the sex and breed of the FIPV-diagnosed cats did not clearly influence the risk of infection. Nevertheless, previous studies have shown that sex, age, and breed affect FIP development [49-51].

In addition to body effusion specimens, we also identified FCoVs from plasma and feces. A previous study showed that FIPV was not only found in the tissue but also detected in oral or conjunctiva samples and feces before death [21, 22, 52].

The current study has several limitations. First, we were not able to obtain complete sequences from all of the samples. This may have been due to high variability in the regions examined. Second, there is a lack of available FCoV accessory protein sequences from previous studies in Thailand, and therefore, an analysis of genetic relationships and genetic evolution of current and historical Thai FCoV strains could not be performed. Third, the cats in this study were from the Bangkok metropolitan region, and disease surveillance in other parts of the country was not included. Fourth, the $\mathrm{S}$ gene was not included in our sequence analysis, and therefore, the FCoV biotype information is still incomplete. Fifth, this study did not include direct detection of FCoV antigen in affected tissue (FIP monoclonal antibody staining) or serological evidence of FCoV infection.

\section{Conclusion}

Samples collected at different times may show the trend of FCoV genotype diversity within the Bangkok metropolitan region. Analysis of accessory gene sequences indicated that Thai FCoVs are more closely related to global FCoVs than to prototypic or vaccine strains. Based on ORF3a and ORF3b sequences, Thai FCoVs were divided into two distinct clusters (G1 and G2). Members of the Thai G2 group shared greater than $99 \%$ sequence identity with the DF-2 prototype and were found to contain premature stop codons. The ORF3c sequence of Thai FCoVs showed the most genetic variation. Amino acid deletions were found in the N, ORF3c, and ORF7b proteins of Thai FCoVs. Nevertheless, we were unable to make an accurate determination of the prevalence of FCoV in Thailand. More sample collection and whole-genome analysis are required to provide the additional sequence information and needed to identify additional field strains that are relevant for further vaccine development.

Supplementary information The online version contains supplementary material available at https://doi.org/10.1007/s00705-021-05188-7.

Acknowledgements We thank Dr. Wassamon Moyadee, staff of the Veterinary Teaching Hospital, Kasetsart University, and Mr. Phanthawat Sakkaew for helpful support and assistance.

Funding This research was supported by a Postdoctoral Fellowship from Kasetsart University Research and Development Institute, Kasetsart University, Bangkok, Thailand, the Faculty of Veterinary Medicine, Kasetsart University, and the Faculty of Science, Kasetsart University, Bangkok Thailand. 


\section{Declarations}

Conflict of interest The authors have no completing of interest to declare.

Ethical approval and consent to participate The research followed the guidelines of Ethical Principles and the Use of Animals for Scientific Purposes from The National Research Council of Thailand. The protocol was approved by the Animal Care and Use Committee (IACUC) (animal use protocol number ACKU64-VET-009) of Kasetsart University, Bangkok, Thailand.

Informed consent Informed consent was obtained from cat owners for using animals in the study.

Availability of data and materials The datasets produced and/or analyzed in the current study are available from the corresponding author on reasonable request.

\section{References}

1. Bredenbeek PJ, Pachuk CJ, Noten AF, Charite J, Luytjes W, Weiss SR, Spaan WJ (1990) The primary structure and expression of the second open reading frame of the polymerase gene of the coronavirus MHV-A59; a highly conserved polymerase is expressed by an efficient ribosomal frameshifting mechanism. Nucleic Acids Res 18:1825-1832. https://doi.org/10.1093/nar/18.7.1825

2. Belouzard S, Millet JK, Licitra BN, Whittaker GR (2012) Mechanisms of coronavirus cell entry mediated by the viral spike protein. Viruses 4:1011-1033. https://doi.org/10.3390/v4061011

3. Licitra BN, Millet JK, Regan AD, Hamilton BS, Rinaldi VD, Duhamel GE, Whittaker GR (2013) Mutation in spike protein cleavage site and pathogenesis of feline coronavirus. Emerg Infect Dis 19:1066-1073. https://doi.org/10.3201/eid1907.121094

4. Dye C, Siddell SG (2005) Genomic RNA sequence of feline coronavirus strain FIPV WSU-79/1146. J Gen Virol 86:2249-2253. https://doi.org/10.1099/vir.0.80985-0

5. Dedeurwaerder A, Desmarets LM, Olyslaegers DAJ, Vermeulen BL, Dewerchin HL, Nauwynck HJ (2013) The role of accessory proteins in the replication of feline infectious peritonitis virus in peripheral blood monocytes. Vet Microbiol 162:447-455. https:// doi.org/10.1016/j.vetmic.2012.10.032

6. Dedeurwaerder A, Olyslaegers DAJ, Desmarets LMB, Roukaerts IDM, Theuns S, Nauwynck HJ (2014) ORF7-encoded accessory protein $7 \mathrm{a}$ of feline infectious peritonitis virus as a counteragent against IFN-alpha-induced antiviral response. J Gen Virol 95:393402. https://doi.org/10.1099/vir.0.058743-0

7. Haijema BJ, Volders H, Rottier PJ (2004) Live, attenuated coronavirus vaccines through the directed deletion of group-specific genes provide protection against feline infectious peritonitis. J Virol 78:3863-3871. https://doi.org/10.1128/jvi.78.8.3863-3871. 2004

8. Herrewegh AA, Vennema H, Horzinek MC, Rottier PJ, de Groot RJ (1995) The molecular genetics of feline coronaviruses: comparative sequence analysis of the ORF7a/7b transcription unit of different biotypes. Virology 212:622-631. https://doi.org/10.1006/ viro.1995.1520

9. Kennedy M, Boedeker N, Gibbs P, Kania S (2001) Deletions in the $7 \mathrm{a}$ ORF of feline coronavirus associated with an epidemic of feline infectious peritonitis. Vet Microbiol 81:227-234. https:// doi.org/10.1016/s0378-1135(01)00354-6
10. Dye C, Siddell SG (2007) Genomic RNA sequence of feline coronavirus strain FCoV C1Je. J Feline Med Surg 9:202-213. https:// doi.org/10.1016/j.jfms.2006.12.002

11. Le Poder S (2011) Feline and canine coronaviruses: common genetic and pathobiological features. Adv Virol 2011:609465. https://doi.org/10.1155/2011/609465

12. Addie DD, Schaap IAT, Nicolson L, Jarrett O (2003) Persistence and transmission of natural type I feline coronavirus infection. J Gen Virol 84:2735-2744. https://doi.org/10.1099/vir.0.19129-0

13. Benetka V, Kubber-Heiss A, Kolodziejek J, Nowotny N, Hofmann-Parisot M, Mostl K (2004) Prevalence of feline coronavirus types I and II in cats with histopathologically verified feline infectious peritonitis. Vet Microbiol 99:31-42. https://doi.org/ 10.1016/j.vetmic.2003.07.010

14. Herrewegh AA, Smeenk I, Horzinek MC, Rottier PJ, de Groot RJ (1998) Feline coronavirus type II strains 79-1683 and 79-1146 originate from a double recombination between feline coronavirus type I and canine coronavirus. J Virol 72:4508-4514

15. Shiba N, Maeda K, Kato H, Mochizuki M, Iwata H (2007) Differentiation of feline coronavirus type I and II infections by virus neutralization test. Vet Microbiol 124:348-352. https://doi.org/ 10.1016/j.vetmic.2007.04.031

16. Kipar A, Meli ML (2014) Feline infectious peritonitis: still an enigma? Vet Pathol 51:505-526. https://doi.org/10.1177/03009 85814522077

17. Porter E, Tasker S, Day MJ, Harley R, Kipar A, Siddell SG, Helps CR (2014) Amino acid changes in the spike protein of feline coronavirus correlate with systemic spread of virus from the intestine and not with feline infectious peritonitis. Vet Res 45:49. https:// doi.org/10.1186/1297-9716-45-49

18. Rottier PJ, Nakamura K, Schellen P, Volders H, Haijema BJ (2005) Acquisition of macrophage tropism during the pathogenesis of feline infectious peritonitis is determined by mutations in the feline coronavirus spike protein. J Virol 79:14122-14130. https://doi.org/10.1128/JVI.79.22.14122-14130.2005

19. Vennema H, Poland A, Foley J, Pedersen NC (1998) Feline infectious peritonitis viruses arise by mutation from endemic feline enteric coronaviruses. Virology 243:150-157. https://doi.org/10. 1006/viro.1998.9045

20. An DJ, Jeoung HY, Jeong W, Park JY, Lee MH, Park BK (2011) Prevalence of Korean cats with natural feline coronavirus infections. Virol J 8:455. https://doi.org/10.1186/1743-422X-8-455

21. Herrewegh AA, de Groot RJ, Cepica A, Egberink HF, Horzinek MC, Rottier PJ (1995) Detection of feline coronavirus RNA in feces, tissues, and body fluids of naturally infected cats by reverse transcriptase PCR. J Clin Microbiol 33:684-689. https://doi.org/ 10.1128/JCM.33.3.684-689.1995

22. Pedersen NC, Liu H, Dodd KA, Pesavento PA (2009) Significance of coronavirus mutants in feces and diseased tissues of cats suffering from feline infectious peritonitis. Viruses 1:166-184. https:// doi.org/10.3390/v1020166

23. Brown MA, Troyer JL, Pecon-Slattery J, Roelke ME, O'Brien SJ (2009) Genetics and pathogenesis of feline infectious peritonitis virus. Emerg Infect Dis 15:1445-1452. https://doi.org/10.3201/ eid1509.081573

24. Pedersen NC, Liu H, Scarlett J, Leutenegger CM, Golovko L, Kennedy H, Kamal FM (2012) Feline infectious peritonitis: role of the feline coronavirus $3 \mathrm{c}$ gene in intestinal tropism and pathogenicity based upon isolates from resident and adopted shelter cats. Virus Res 165:17-28. https://doi.org/10.1016/j.virusres. 2011.12.020

25. Vennema H (1999) Genetic drift and genetic shift during feline coronavirus evolution. Vet Microbiol 69:139-141. https://doi.org/ $10.1016 / \mathrm{s} 0378-1135(99) 00102-9$ 
26. Tasker S (2018) Diagnosis of feline infectious peritonitis: update on evidence supporting available tests. J Feline Med Surg 20:228 243. https://doi.org/10.1177/1098612X18758592

27. Amer A, Siti Suri A, Abdul Rahman O, Mohd HB, Faruku B, Saeed S, Tengku Azmi TI (2012) Isolation and molecular characterization of type I and type II feline coronavirus in Malaysia. Virol J 9:278. https://doi.org/10.1186/1743-422X-9-278

28. Tamura K, Stecher G, Peterson D, Filipski A, Kumar S (2013) MEGA6: molecular evolutionary genetics analysis version 6.0. Mol Biol Evol 30:2725-2729. https://doi.org/10.1093/molbev/ mst197

29. Hartmann K, Binder C, Hirschberger J, Cole D, Reinacher M, Schroo S, Frost J, Egberink H, Lutz H, Hermanns W (2003) Comparison of different tests to diagnose feline infectious peritonitis. J Vet Intern Med 17:781-790. https://doi.org/10.1111/j.1939-1676. 2003.tb02515.x

30. Hazuchova K, Held S, Neiger R (2017) Usefulness of acute phase proteins in differentiating between feline infectious peritonitis and other diseases in cats with body cavity effusions. J Feline Med Surg 19:809-816. https://doi.org/10.1177/1098612X16658925

31. Pedersen NC, Eckstrand C, Liu H, Leutenegger C, Murphy B (2015) Levels of feline infectious peritonitis virus in blood, effusions, and various tissues and the role of lymphopenia in disease outcome following experimental infection. Vet Microbiol 175:157-166. https://doi.org/10.1016/j.vetmic.2014.10.025

32. Stranieri A, Giordano A, Paltrinieri S, Giudice C, Cannito V, Lauzi S (2018) Comparison of the performance of laboratory tests in the diagnosis of feline infectious peritonitis. J Vet Diagn Investig 30:459-463. https://doi.org/10.1177/1040638718756460

33. Moyadee W, Jaroensong T, Roytrakul S, Boonkaewwan C, Rattanasrisomporn J (2019) Characteristic clinical signs and blood parameters in cats with Feline Infectious Peritonitis. Agric Nat Resour 53:433-438. https://doi.org/10.34044/j.anres.2019.53.4. 15

34. Jeffery U, Deitz K, Hostetter S (2012) Positive predictive value of albumin: globulin ratio for feline infectious peritonitis in a midwestern referral hospital population. J Feline Med Surg 14:903905. https://doi.org/10.1177/1098612X12454862

35. Giori L, Giordano A, Giudice C, Grieco V, Paltrinieri S (2011) Performances of different diagnostic tests for feline infectious peritonitis in challenging clinical cases. J Small Anim Pract 52:152-157. https://doi.org/10.1111/j.1748-5827.2011.01042.x

36. Yin Y, Li T, Wang C, Liu X, Ouyang H, Ji W, Liu J, Liao X, Li $\mathrm{J}, \mathrm{Hu} \mathrm{C}$ (2021) A retrospective study of clinical and laboratory features and treatment on cats highly suspected of feline infectious peritonitis in Wuhan, China. Sci Rep 11:5208. https://doi.org/10. 1038/s41598-021-84754-0

37. Techangamsuwan S, Radtanakatikanon A, Purnaveja S (1970) Molecular detection and genotype differentiation of feline coronavirus isolates from clinical specimens in Thailand. Thai J Vet Med 42:413-422

38. Landes C, Kriegleder H, Lengfelder KD (1984) Causes of death and disease in cats based on 1969-1982 autopsy statistics. Tierarztl Prax 12:369-382

39. Holzworth J (1963) Infectious diseases of cats. Cornell Vet 53:131-143

40. Lauzi S, Stranieri A, Giordano A, Luzzago C, Zehender G, Paltrinieri S, Ebranati E (2020) Origin and transmission of Feline coronavirus type I in domestic cats from Northern Italy: a phylogeographic approach. Vet Microbiol 244:108667. https://doi.org/ 10.1016/j.vetmic.2020.108667
41. Pedersen NC (2009) A review of feline infectious peritonitis virus infection: 1963-2008. J Feline Med Surg 11:225-258. https://doi. org/10.1016/j.jfms.2008.09.008

42. Balint A, Farsang A, Zadori Z, Belak S (2014) Comparative in vivo analysis of recombinant type II feline coronaviruses with truncated and completed ORF3 region. PLoS ONE 9:e88758. https://doi.org/10.1371/journal.pone.0088758

43. Balint A, Farsang A, Zadori Z, Hornyak A, Dencso L, Almazan F, Enjuanes L, Belak S (2012) Molecular characterization of feline infectious peritonitis virus strain DF-2 and studies of the role of ORF3abc in viral cell tropism. J Virol 86:6258-6267. https://doi. org/10.1128/JVI.00189-12

44. Chang HW, de Groot RJ, Egberink HF, Rottier PJ (2010) Feline infectious peritonitis: insights into feline coronavirus pathobiogenesis and epidemiology based on genetic analysis of the viral 3c gene. J Gen Virol 91:415-420. https://doi.org/10.1099/vir.0. 016485-0

45. Lin CN, Su BL, Huang HP, Lee JJ, Hsieh MW, Chueh LL (2009) Field strain feline coronaviruses with small deletions in ORF7b associated with both enteric infection and feline infectious peritonitis. J Feline Med Surg 11:413-419. https://doi.org/10.1016/j. jfms.2008.09.004

46. Bank-Wolf BR, Stallkamp I, Wiese S, Moritz A, Tekes G, Thiel HJ (2014) Mutations of 3c and spike protein genes correlate with the occurrence of feline infectious peritonitis. Vet Microbiol 173:177-188. https://doi.org/10.1016/j.vetmic.2014.07.020

47. Lutz M, Steiner AR, Cattori V, Hofmann-Lehmann R, Lutz H, Kipar A, Meli ML (2020) FCoV viral sequences of systemically infected healthy cats lack gene mutations previously linked to the development of FIP. Pathogens. https://doi.org/10.3390/patho gens 9080603

48. Takano T, Tomiyama Y, Katoh Y, Nakamura M, Satoh R, Hohdatsu T (2011) Mutation of neutralizing/antibody-dependent enhancing epitope on spike protein and $7 \mathrm{~b}$ gene of feline infectious peritonitis virus: influences of viral replication in monocytes/macrophages and virulence in cats. Virus Res 156:72-80. https://doi.org/10.1016/j.virusres.2010.12.020

49. Pedersen NC, Liu H, Gandolfi B, Lyons LA (2014) The influence of age and genetics on natural resistance to experimentally induced feline infectious peritonitis. Vet Immunol Immunopathol 162:33-40. https://doi.org/10.1016/j.vetimm.2014.09.001

50. Soma T, Wada M, Taharaguchi S, Tajima T (2013) Detection of ascitic feline coronavirus RNA from cats with clinically suspected feline infectious peritonitis. J Vet Med Sci 75:1389-1392. https:// doi.org/10.1292/jvms.13-0094

51. Worthing KA, Wigney DI, Dhand NK, Fawcett A, McDonagh P, Malik R, Norris JM (2012) Risk factors for feline infectious peritonitis in Australian cats. J Feline Med Surg 14:405-412. https:// doi.org/10.1177/1098612X12441875

52. Wang YT, Su BL, Hsieh LE, Chueh LL (2013) An outbreak of feline infectious peritonitis in a Taiwanese shelter: epidemiologic and molecular evidence for horizontal transmission of a novel type II feline coronavirus. Vet Res 44:57. https://doi.org/10.1186/ 1297-9716-44-57

Publisher's Note Springer Nature remains neutral with regard to jurisdictional claims in published maps and institutional affiliations. 